\title{
CHARACTERIZATIONS OF SIZE EFFECT AND OVERALL MECHANICAL BEHAVIOR OF NANOCRYSTALLINE METALS
}

\author{
LI CHEN* and YUEGUANG WEI $^{\dagger}$ \\ State Key Laboratory of Nonlinear Mechanics \\ Institute of Mechanics, Chinese Academy of Sciences \\ Beijing 100190, P. R. China \\ *cchenli@Lnm.imech.ac.cn \\ †ywei@Lnm.imech.ac.cn
}

Received 21 October 2012

Accepted 22 December 2012

Published 27 March 2013

\begin{abstract}
A systematical study of size effects and mechanical behaviors for the nanocrystalline (nc) metals is performed. The grain boundary fracture process is considered and described by the mixed-mode interface cohesive model. The grain material is characterized by the conventional theory of strain gradient plasticity. In the present investigation, the effects of five important parameters on the overall mechanical behavior are studied systematically, which include the grain size, critical separation strength, energy release rate of interface separation, mixity of separation strength, as well as the mixity of separation energy release rate. A finite element method (FEM) covering the above characteristics within the grain and on the grain boundary is developed. The present results show that the overall strength and ductility of the nc metals strongly depend on the grain boundary features described by the mixed-mode cohesive interface model, and there is a competition of deformation of grain boundary with that of grain interior.
\end{abstract}

Keywords: Intergranular fracture; nanocrystalline metal; mixed-mode cohesive model; finite element method.

\section{Introduction}

Mechanical behaviors of polycrystalline materials with grain sizes typically at nanoor micron scale (such as nanocrystalline (nc) metals) have been attracting a great deal of interest over the past two decades. The nc metals exhibit the higher yield strength, tensile strength and hardness, but the lower tensile ductility relative to their bulk counterparts. Although some mechanisms have been presented for governing the mechanical behavior of polycrystalline aggregates [Gleiter, 2000; Kumar et al., 2003; Wei et al., 2005], very few direct experimental evidences exist to show the fracture and failure processes in the nc metals, especially inelastic deformation competition of grain interior and grain boundary. Shan et al. [2004] reported that 
the grain boundary-mediated process of the nc nickel film dominates its deformation in the observation using the transmission electron microscope (TEM). Moreover, the molecular dynamics (MD) simulations have shown that grain boundary related slip and separation phenomena plays an important role in the overall inelastic response with decreasing grain-size [Cao and Wei, 2007; Hasnaoui et al., 2003; Schiotz et al., 1999; Van Swygenhoven and Derlet, 2001; Van Vliet et al., 2003]. Due to the limitation of time and dimensional scales in MD simulations for the mechanical behaviors of the nc metals with realistic experiment sample sizes and strain rates, several continuum models have been used to describe the grain boundary effect and the failure response for the nc materials [Fu et al., 2004; Ovid'ko, 2007; Schwaiger et al., 2003; Warner et al., 2006; Wei et al., 2005, 2006; Wei and Anand, 2004]. Considering the inherently characteristics of grain boundaries in the nc metals, both the grain boundary affected zone (GBAZ) model [Fu et al., 2004; Schwaiger et al., 2003; Wei et al., 2005, 2006] and the cohesive interface model [Warner et al., 2006; Wei and Anand, 2004] were proposed to characterize the grain boundary response in polycrystalline aggregates.

Specifically, Wu and Wei [2010] studied the size effects of mechanical behavior for the nc metals by adopting the cohesive interface model and the conventional theory of mechanism-based strain gradient plasticity (CMSG) [Huang et al., 2004], and they used a normal separation cohesive model to describe the grain boundary separation. More recently, $\mathrm{Wu}$ et al. [2012] investigated the size effects of mechanical behavior for the nc metals. They presented a trans-scale mechanics theory with respect to both the interface energy effect and the strain gradient effect, and they used the normal separation cohesive model to describe the grain boundary fracture process.

Although the comprehensive analyses of mechanical behaviors for the nc materials by using above-mentioned continuum models and the normal separation cohesive interface model were carried out recently, it is still difficult to unambiguously define the interfacial properties of grain boundaries. The conventional elastic-plastic theory is also difficult to characterize the size effects of intragranular deformation in nanoscale. Therefore, in the present research, we shall pay our attention in using a mixed-mode cohesive interface model to describe the grain boundary deformation, separation as well as the fracture. In addition, the CMSG model [Huang et al., 2004] will be used to characterize the strain hardening of grain materials. Special attention is focused on the effects of grain boundary properties and grain sizes on the size effect and overall strength and ductility of nc metals.

\section{Descriptions of Overall Mechanical Behavior for nc Materials}

\subsection{Grain material}

When grain size is at micro- or nanoscale, the mechanical behavior of the grain material cannot be well described by using the classical elastic-plastic theory. So in the present research, the mechanical behavior of the grain material is described by 
the strain gradient theory. For simplifying the analysis, the CMSG theory [Huang et al., 2004] will be used. Unlike the general strain gradient plasticity theory [Gao et al., 1999; Wei and Hutchinson, 1997a], the CMSG theory is a lower-order theory which does not involve the higher-order stress, and is easy to develop a finite element scheme.

Briefly, the constitutive relation for CMSG theory [Huang et al., 2004] can be expressed as follows:

$$
\dot{\sigma}_{i j}=K \dot{\varepsilon}_{k k} \delta_{i j}+2 \mu\left\{\dot{\varepsilon}_{i j}^{\prime}-\frac{3 \dot{\varepsilon}}{2 \sigma_{e}}\left(\frac{\sigma_{e}}{\sigma_{\mathrm{Y}} \sqrt{f^{2}\left(\varepsilon^{p}\right)+l \eta^{p}}}\right)^{m} \sigma_{i j}^{\prime}\right\},
$$

where $K$ is the bulk modulus, $\mu$ is the shear modulus, $m$ is the rate-sensitivity exponent $(m \geq 20)$ [Hutchinson, 1976; Kok et al., 2002], $\varepsilon^{\mathrm{p}}$ and $\eta^{\mathrm{p}}$ are the effective plastic strain and plastic strain gradient, which can be expressed as:

$$
\begin{aligned}
& \eta^{\mathrm{p}}=\sqrt{\frac{1}{4} \eta_{i j k}^{\mathrm{p}} \eta_{i j k}^{\mathrm{p}}}, \quad \eta_{i j k}^{\mathrm{p}}=\varepsilon_{i k, j}^{\mathrm{p}}+\varepsilon_{j k, i}^{\mathrm{p}}-\varepsilon_{i j, k}^{\mathrm{p}}, \\
& \varepsilon_{i j}^{\mathrm{p}}=\int \dot{\varepsilon}_{i j}^{\mathrm{p}} d t, \quad \varepsilon^{\mathrm{p}}=\sqrt{\frac{2}{3} \varepsilon_{i j}^{\mathrm{p}} \varepsilon_{i j}^{\mathrm{p}}},
\end{aligned}
$$

$l$ is the intrinsic material length scale in strain gradient plasticity, $\sigma_{\mathrm{Y}}$ is the initial yield stress, $f$ is a non-dimensional function of plastic strain which takes the form as follows for a power-law hardening solid,

$$
f\left(\varepsilon^{\mathrm{p}}\right)=\left(1+\frac{E \varepsilon^{\mathrm{p}}}{\sigma_{\mathrm{Y}}}\right)^{N}
$$

where $E$ is the Young's modulus, $N$ is the strain hardening exponent $(0 \leq N<1)$. $\dot{\varepsilon}=\sqrt{2 / 3 \dot{\varepsilon}_{i j}^{\prime} \dot{\varepsilon}_{i j}^{\prime}}$ is the effective strain rate, $\dot{\varepsilon}_{i j}^{\prime}$ is the deviatoric strain rate and $\sigma_{e}=$ $\sqrt{3 \sigma_{i j}^{\prime} \sigma_{i j}^{\prime} / 2}$ is the von Mises effective stress.

\subsection{Grain boundary description}

Grain boundary fracture process is simulated by using the cohesive interface model, which was performed early by Barenblatt [1959, 1962] and Dugdale [1960]. In last several decades, the cohesive interface models have undergone a great development in describing the materials fracture process by using the finite element methods (FEMs) [Needleman, 1990; Tvergaard and Hutchinson, 1992; Wei and Hutchinson, 1997b]. The traction-separation (T-S) relations of cohesive interface have been extended to represent the fracture process of grain boundaries [Warner et al., 2006; Wei and Anand, 2004; Xu et al., 2010]. In the present investigation, a mixed-mode cohesive interface model developed by Turon et al. [2004] will be used to describe the initiation and evolution of grain boundary fracture.

As is well known, keeping both height and area, shape effect of $\mathrm{T}-\mathrm{S}$ cohesive relation on the material behavior is small and can be neglected usually. Therefore, for simplification we shall use a bilinear mixed-mode cohesive model in the present 


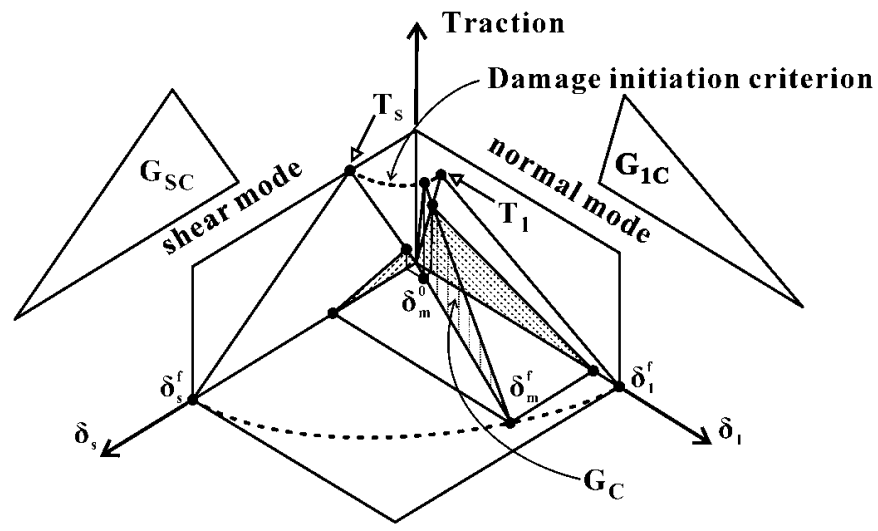

(a)

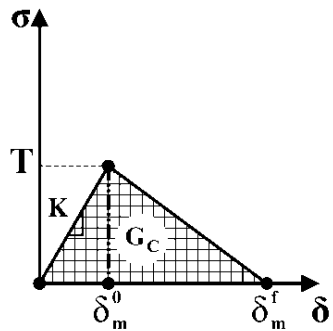

Total Traction-Separation response

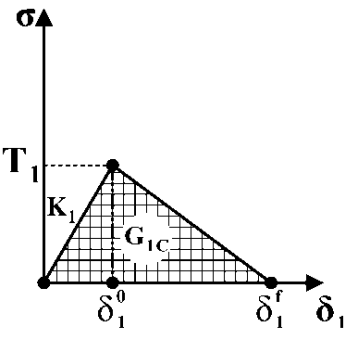

Pure I mode

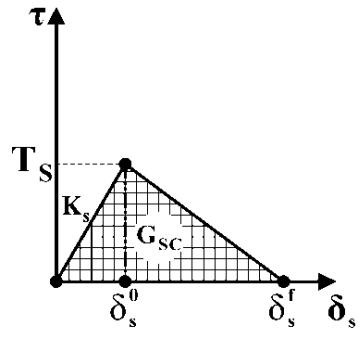

Shear mode

(b)

Fig. 1. Illustration of mixed-mode cohesive interface model (a) and bilinear T-S response (b).

study, which is pictured in Fig. 1 in detail in a 3D map. Three coordinate axes are normal relative displacement $\delta_{1}$, shearing relative displacement $\delta_{\mathrm{s}}$ and traction, respectively. Figure 1 shows the scheme of the mixed-mode cohesive interface model within the space of both traction and separation displacements, and shows the relationship between the mixed-mode cohesive model with the pure normal separation cohesive model as well as with the pure shear separation cohesive model. Referring to Fig. 1, both the triangle $O-T_{1}-\delta_{1}^{\mathrm{f}}$ and $O-T_{\mathrm{S}}-\delta_{\mathrm{s}}^{\mathrm{f}}(O$ is coordinate origin) are the bilinear responses in pure normal and pure shear modes, respectively. Any point located on the $O-\delta_{\mathrm{s}}^{\mathrm{f}}-\delta_{1}^{\mathrm{f}}$ plane will correspond to a mixed-mode interface separation process. Subscripts "1" and "s" are used to represent the pure normal mode and pure shear mode, respectively. The critical relative displacements corresponding to the initiation of damage are identified with the superscript " 0 ". The limit relative displacements corresponding to failure state are identified with the superscript "f".

The relations between critical relative displacements and maximum tractions are given by:

$$
\delta_{1}^{0}=\frac{T_{1}}{K_{1}}, \quad \delta_{\mathrm{s}}^{0}=\frac{T_{\mathrm{S}}}{K_{\mathrm{S}}}
$$


where $T_{1}$ and $T_{\mathrm{S}}$ are the limit tractions for pure normal mode and pure shear mode, respectively. $K_{1}$ and $K_{\mathrm{S}}$ are the penalty stiffness.

The relations between the maximum relative displacements and fracture energies can be written as follows:

$$
\delta_{1}^{\mathrm{f}}=\frac{2 G_{1 \mathrm{C}}}{K_{1} \delta_{1}^{0}}, \quad \delta_{\mathrm{s}}^{\mathrm{f}}=\frac{2 G_{\mathrm{SC}}}{K_{\mathrm{S}} \delta_{\mathrm{S}}^{0}},
$$

where $G_{1 \mathrm{C}}$ and $G_{\mathrm{SC}}$ are the fracture energies for pure normal mode and pure shear mode, respectively.

In order to describe the combination effect of normal and shear deformations across the interface, an effective relative displacement is introduced as follows:

$$
\delta_{\mathrm{m}}=\sqrt{\left\langle\delta_{1}\right\rangle^{2}+\delta_{\mathrm{s}}^{2}}
$$

where symbol $\left\langle\delta_{1}\right\rangle$ is Macaulay bracket of $\delta_{1}$, which signifies that its value is equal to zero for $\delta_{1} \leq 0$, otherwise $\delta_{1}$, where $\delta_{1}$ and $\delta_{\mathrm{s}}$ are the normal separation and shear relative displacements along the interface, respectively.

The mixed-mode T-S relations are illustrated in Fig. 1(b), where $\sigma$ is the traction, $\delta_{\mathrm{m}}^{0}$ is the critical effective separation displacement at damage initiation, $T$ is the critical traction and $\delta_{\mathrm{m}}^{\mathrm{f}}$ is effective separation displacement at failure state. Both $\delta_{\mathrm{m}}^{0}$ and $\delta_{\mathrm{m}}^{\mathrm{f}}$ are defined by the mixed-mode damage initiation and fracture criteria as determined according to the following Eqs. (7) and (8), respectively.

The damage is assumed to be initiated when the following quadratic relation is satisfied [Mi et al., 1998]:

$$
\left(\frac{\left\langle\sigma_{1}\right\rangle}{T_{1}}\right)^{2}+\left(\frac{\tau_{\mathrm{s}}}{T_{\mathrm{S}}}\right)^{2}=1,
$$

where $\sigma_{1}$ and $\tau_{\mathrm{s}}$ are the normal and shear stresses on the interface, respectively. $T_{1}$ and $T_{\mathrm{S}}$ are the limit tractions for pure normal mode and pure shear mode, respectively.

The mixed-mode fracture criterion is described as follows [Mi et al., 1998]:

$$
\frac{G_{1}}{G_{1 \mathrm{C}}}+\frac{G_{\mathrm{S}}}{G_{\mathrm{SC}}}=1,
$$

where $G_{1}$ and $G_{\mathrm{S}}$ are the current fracture energies for the normal and shear cases, respectively, $G_{\mathrm{C}}=G_{1}+G_{\mathrm{S}}$ is the total current fracture energy for mixed-mode case when above condition is satisfied.

\section{Numerical Simulations of Mechanical Behavior of the nc Materials}

\subsection{Cell model and periodic boundary conditions}

A regular quasi-three-dimensional cell model is presented here. Figure 2 shows the schematic drawing of the cell model. The cell model is consisted of 12 regular hexagon grains and the grain size $d$ is the diameter of the circumcircle of regular 


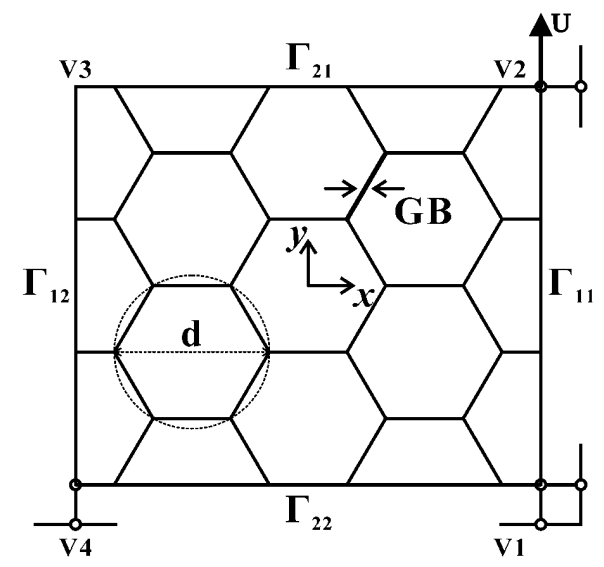

Fig. 2. A representative cell model for nc metal with periodic boundary conditions of $x y$-plane and $z$-direction.

hexagon. The separation process of grain boundary between two hexagon grains is modeled by using a single layer of the cohesive elements with zero thickness, as shown in Fig. 2.

Generally speaking, nc materials can be envisaged as a periodical array of the cell model, therefore, the periodic boundary conditions can be applied. As displayed in Fig. 2, periodic boundary conditions are enforced along the four sides in $x y$ coordinate plane [Van der Sluis et al., 2001]:

$$
\begin{aligned}
& \overrightarrow{\mathbf{u}}_{12}-\overrightarrow{\mathbf{u}}_{\mathrm{V} 4}=\overrightarrow{\mathbf{u}}_{11}-\overrightarrow{\mathbf{u}}_{\mathrm{V} 1}, \\
& \overrightarrow{\mathbf{u}}_{22}-\overrightarrow{\mathbf{u}}_{\mathrm{V} 1}=\overrightarrow{\mathbf{u}}_{21}-\overrightarrow{\mathbf{u}}_{\mathrm{V} 2}, \\
& \overrightarrow{\mathbf{u}}_{\mathrm{V} 3}-\overrightarrow{\mathbf{u}}_{\mathrm{V} 2}=\overrightarrow{\mathbf{u}}_{\mathrm{V} 4}-\overrightarrow{\mathbf{u}}_{\mathrm{V} 1},
\end{aligned}
$$

where $\overrightarrow{\mathbf{u}}_{\mathrm{ij}}$ is a displacement vector for a material point on the boundary $\Gamma_{\mathrm{ij}}$, and $\overrightarrow{\mathbf{u}}_{\mathrm{Vi}}$ is a displacement vector for the vertex Vi. In Eqs. (9)-(11), both the periodic boundary conditions and the rigid displacement eliminating conditions are satisfied simultaneously.

An arbitrary periodically deformed unit cell under uniaxial tensile conditions is shown in Fig. 3, where (a) represents the initial undeformed state and (b) represents the deformed state under periodic boundary conditions.

If the nc material is treated as a plane-strain case, the strength of the material will be over-estimated, in order to describe the three-dimensional effect, in the present research a quasi-three-dimensional case will be considered which corresponds to exerting a periodic boundary condition in $z$ direction. A displacement vector $\overrightarrow{\mathbf{u}}_{z}$ on boundary is enforced in the $z$-coordinate direction, assuming that the material geometry in $z$-direction is also a periodic structure which has a finite-thickness [Wu and Wei, 2010]. 

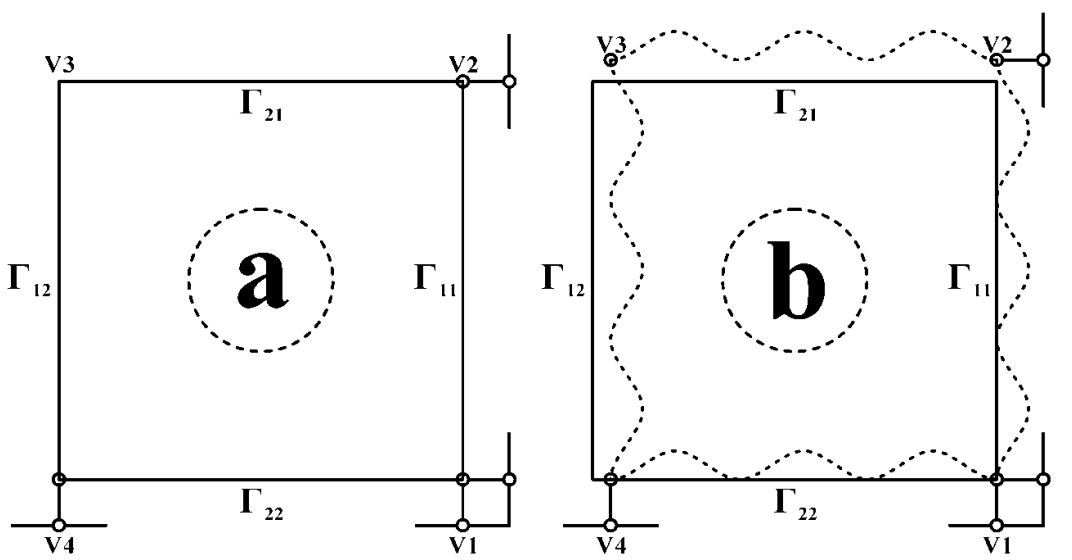

Fig. 3. Illustration of periodic boundary conditions. (a) Represents the initial undeformed state. (b) Represents the deformed state under periodic boundary conditions.

\subsection{Overall mechanical behaviors of nc materials}

The main purpose of the present research is not only to simulate the mechanical behavior of a specific polycrystalline material, but also to provide some insights into the effects of the cohesive parameters on the overall strength and failure behavior of the nc materials. A systematical parametric study will be performed. The overall stress-strain relation normalized by the initial yield stress $\sigma_{\mathrm{Y}}$ and intrinsic material length $l$ with parameter dependence can be expressed as:

$$
\frac{\sigma}{\sigma_{\mathrm{Y}}}=f(\varepsilon ; \underbrace{\frac{E}{\sigma_{\mathrm{Y}}}, v, N, \frac{d}{l}}_{\text {intragranular parameters }}, \underbrace{\frac{T_{1}}{\sigma_{\mathrm{Y}}}, \frac{T_{\mathrm{S}}}{T_{1}}, \frac{G_{1 \mathrm{C}}}{\sigma_{\mathrm{Y}} l}, \frac{G_{\mathrm{SC}}}{G_{1 \mathrm{C}}}, \frac{\bar{K}_{1}}{\sigma_{\mathrm{Y}}} \frac{\bar{K}_{\mathrm{S}}}{\sigma_{\mathrm{Y}}}}_{\text {interfacial parameters }}) .
$$

Specially, there exist two additional important parameters $\left(T_{\mathrm{S}} / T_{1}\right.$ and $\left.G_{\mathrm{SC}} / G_{1 \mathrm{C}}\right)$ compared to conventional cohesive interface model since the mixed-mode cohesive interface model is adopted. Intragranular parameters are Young's modulus $E$, Poisson's ratio $v$, strain hardening exponent $N$ and grain size $d$, respectively. The interfacial parameters of grain boundary are maximum separation strengths $T_{1}$ and $T_{\mathrm{S}}$, fracture energy $G_{1 \mathrm{C}}$ and $G_{\mathrm{SC}}$ for normal separation mode and shear mode, initial separation modulus $\bar{K}_{1}=K_{1} l=T_{1} /\left(\delta_{1}^{0} / l\right), \bar{K}_{\mathrm{S}}=K_{\mathrm{S}} l=T_{\mathrm{S}} /\left(\delta_{\mathrm{s}}^{0} / l\right)$, respectively.

For convenience, we define a mixed angle of the limit traction ratio of pure shear mode with pure normal mode as:

$$
\varphi=\arctan \left(T_{\mathrm{S}} / T_{1}\right) * 180 / \pi,
$$

where $\varphi=0^{\circ}$ and $\varphi=90^{\circ}$ correspond to the normal separation case and shearing separation case, respectively. Similarly, another mixed angle of the limit energy 
release rate ratio of pure shear mode with pure normal mode is defined as:

$$
\Phi=\arctan \left(G_{\mathrm{SC}} / G_{1 \mathrm{C}}\right) * 180 / \pi
$$

where $\Phi=0^{\circ}$ and $\Phi=90^{\circ}$ correspond to the normal separation case and shearing separation case, respectively. So the function relation of stress with strain and with normalized independent parameters (Eq. (12)) can be rewritten as follows.

$$
\frac{\sigma}{\sigma_{\mathrm{Y}}}=g(\varepsilon ; \underbrace{\frac{E}{\sigma_{\mathrm{Y}}}, v, N, \frac{d}{l}}_{\text {intragranular parameters }}, \underbrace{\frac{T_{1}}{\sigma_{\mathrm{Y}}}, \varphi, \frac{G_{1 \mathrm{C}}}{\sigma_{\mathrm{Y}} l}, \Phi, \frac{\bar{K}_{1}}{\sigma_{\mathrm{Y}}} \frac{\bar{K}_{\mathrm{S}}}{\sigma_{\mathrm{Y}}}}_{\text {interfacial parameters }}) .
$$

\subsection{Finite element method}

FEM is used in numerical simulations for overall mechanical behavior of nc materials. Generally speaking, when the strain gradient effect is considered, the conventional FEM fails [Wei, 2006]. The CMSG theory is a lower-order strain gradient theory without involving the higher-order stress, so in this case the governing equation, boundary conditions and algorithms usually employed in classical mechanics are readily available and can be conveniently applied to study problem when strain gradient effect is considered. One can easily modify the existing finite element program to incorporate the plastic strain gradient effect approximately [Swaddiwudhipong et al., 2006]. In the present research, we have implemented a $\mathrm{C}^{0}$

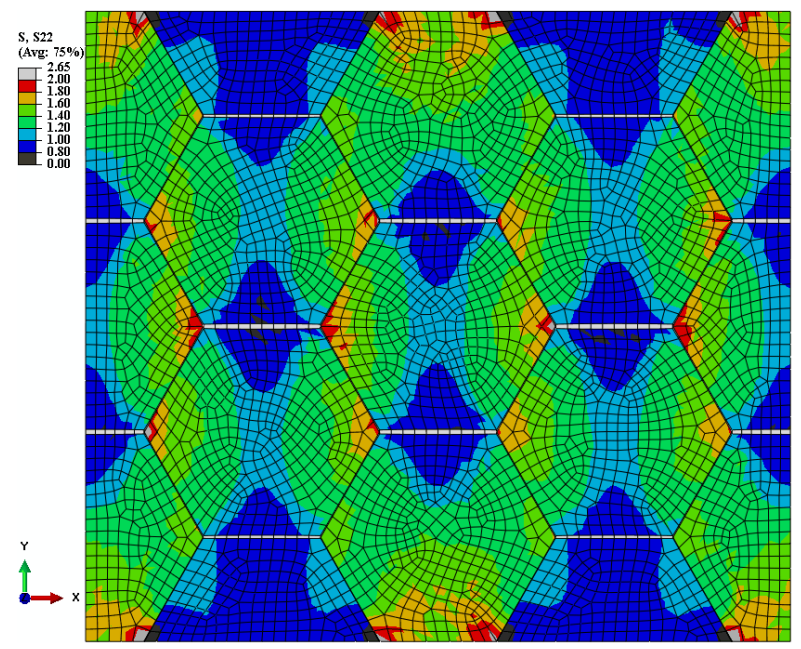

Fig. 4. (Color online) Cloud figure of the normalized stress $\sigma_{22} / \sigma_{\mathrm{Y}}$ (loading direction is along $y$-direction) is shown for $d=0.1 l_{0}, \varphi=45^{\circ}$. Its average value corresponds to overall stress and the peak stress when exerting load reaches maximum value. 
three-dimensional solid element incorporating the CMSG theory in the ABAQUS software via its user subroutine UMAT.

The influences of intragranular and interfacial parameters on the overall mechanical behaviors are investigated by using the FEM. The study is based on a reference parameters selected by the comparison of results between present model and the experimental measurements (in Sec. 4.5). The reference parameters used in the present research are selected as: $E / \sigma_{\mathrm{Y}}=166.6, \nu=0.3, N=0.2$, $T_{\mathrm{S}}=T_{1}=\sigma_{\mathrm{Y}}, G_{\mathrm{SC}}=G_{1 \mathrm{C}}=0.053 \sigma_{\mathrm{Y}} l, l=l_{0}=18 \alpha^{2}\left(\mu / \sigma_{\mathrm{Y}}\right)^{2} b \approx 1 \mu m$ [Huang et al., 2004], where $b$ is magnitude of Burgers vector; and $\alpha$ is an empirical coefficient around 0.3. In the parametric study, $\bar{K}_{1}$ and $\bar{K}_{\mathrm{S}}$ is taken as Young's modulus of crystal grain.

The finite element mesh adopted in the present research can refer to Fig. 4. The grain interior is discredited with hexahedron, eight-node continuum elements C3D8, while the cohesive elements with zero thickness used to simulate grain boundaries are hexahedron, eight-node cohesive elements COH3D8. The element numbers of both C3D8 and COH3D8 for a cell model are 3883 and 342, respectively.

\section{Results and Discussions}

\subsection{Effect of the ratio $T_{\mathrm{s}} / T_{1}$}

Referring to Fig. 2, considering the cell with the periodic boundary conditions under unidirectional tension (in y), vertical normal stress $\sigma_{22} / \sigma_{\mathrm{Y}}$ is calculated first, and the stress cloud figure is given in Fig. 4. Average value of the vertical normal stress is the "overall stress" of cell material, and its limit value is called the "peak stress" in the present research. From the distribution of vertical normal stress in Fig. 4, clearly, there exist three-level loading regions, described by yellow, green and blue colors. The yellow (or red) color region is the high-stress region, while the blue color region is the low-stress region.

The dependence of overall stress-strain relations on the mixed angle $\varphi$ for three selected grain size values (in our simulation, $l=l_{0}=18 \alpha^{2}\left(\mu / \sigma_{\mathrm{Y}}\right)^{2} b=1 \mu \mathrm{m}$ ) is shown in Fig. 5 taking $T_{1}$ as $\sigma_{\mathrm{Y}}$. We consider five cases of $\varphi$ values corresponding to several ratios of $T_{\mathrm{s}} / T_{1}$. From Fig. 5 , the results show that the overall strength and ductility for nc materials are very sensitive to $\varphi$ (describing the ratio $T_{\mathrm{s}} / T_{1}$ ).

In Fig. 6, the curves of the peak stresses (ultimate tensile stress, UTS) with the mixed angle are given, which increase with decreasing grain size, especially for $\varphi \geq 45^{\circ}$, and display obvious size effects.

From Figs. 5 and 7, it is clearly displayed that the ultimate failure strains increase with decreasing $\varphi$ for $\varphi \leq 45^{\circ}$ and the overall stress-strain curves display a ductile fracture feature, which is because the grain boundaries related slip and separation phenomena play more important role in the overall inelastic response of the nc materials when $T_{\mathrm{S}} \leq T_{1}=\sigma_{\mathrm{Y}}$. However, for $\varphi \geq 45^{\circ}$, the ultimate failure strains 


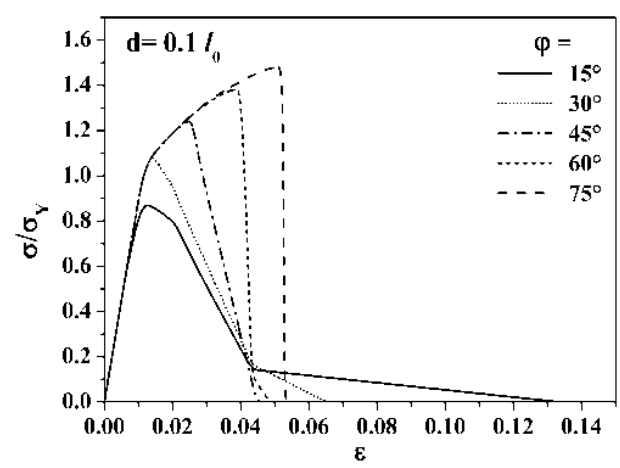

(a)

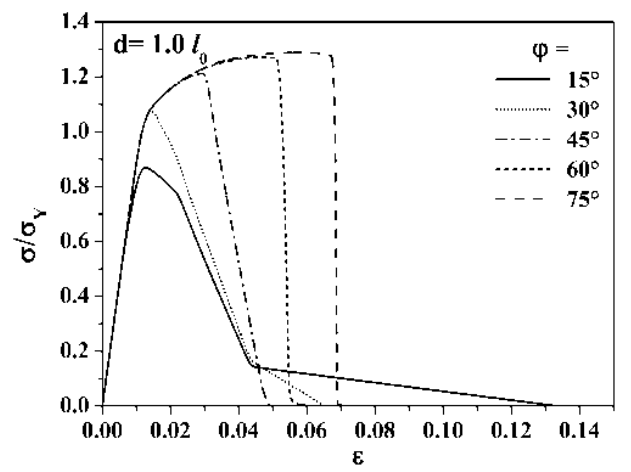

(b)

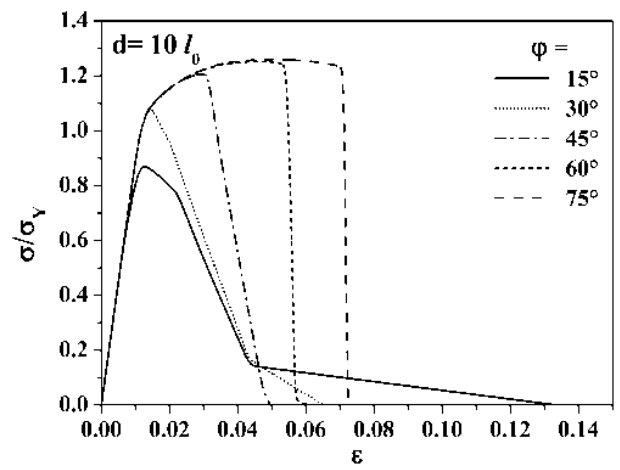

(c)

Fig. 5. Overall stress-strain curves for several values of ratio $T_{\mathrm{s}} / T_{1}$ (see Eq. (13)). (a)-(c) Correspond to three selected grain sizes.

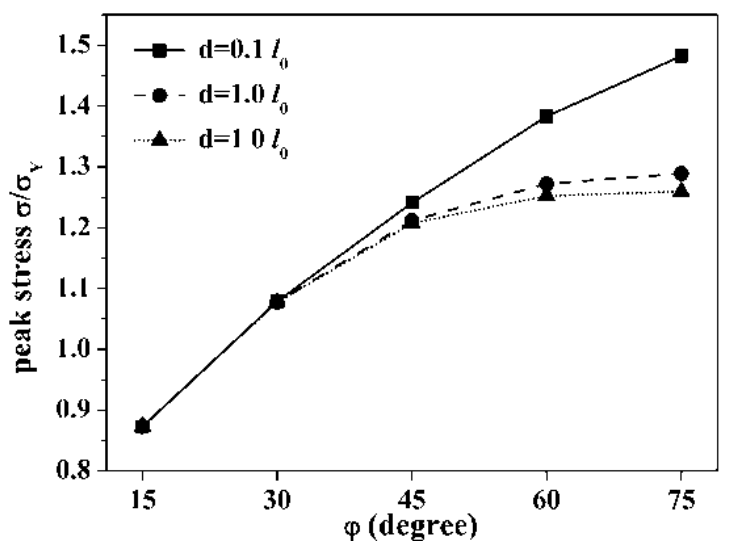

Fig. 6. The dependence of peak stress on the different ratio $T_{\mathrm{S}} / T_{1}$ for three selected grain sizes. 


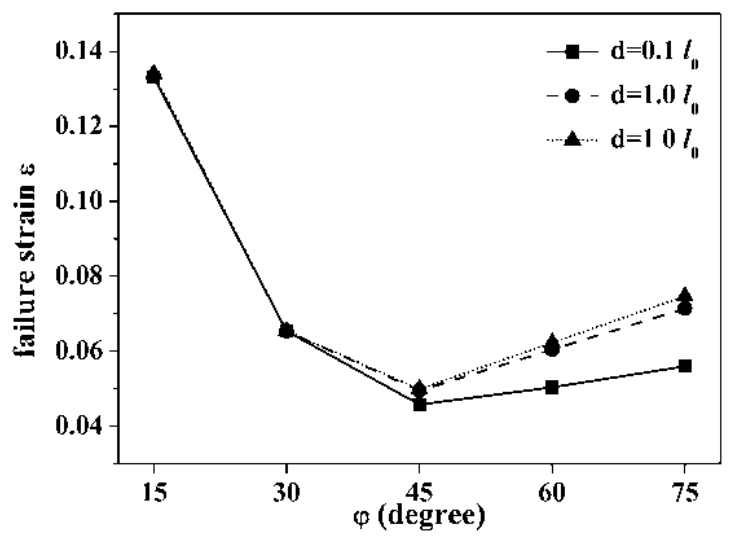

Fig. 7. The dependence of ultimate failure strain on the different ratio $T_{\mathrm{S}} / T_{1}$ for three selected grain sizes.

increase with increasing $\varphi$. The overall stress-strain curves display a brittle fracture feature. Therefore, the intragranular elastic-plastic deformation is dominant and the nc materials display a good ductility and high strength.

\subsection{Effect of the ratio $G_{\mathrm{SC}} / G_{1 \mathrm{C}}$}

The dependence of overall stress-strain relations on the mixed angle $\Phi$ (referring to Eq. (14)) for three selected grain sizes is given in Fig. 8. In the figure, five cases of $\Phi$ values are considered (keeping $G_{1 \mathrm{C}}$ constant). From Figs. 8 and 10, the ductility of nc material is sensitive to $\Phi$ (describing the ratio $G_{\mathrm{SC}} / G_{1 \mathrm{C}}$ ). On the other hand, the overall strength of nc material is not sensitive to the value of $\Phi$ from Figs. 8 and 9 .

\subsection{Effect of the maximum separation strength $T_{1}$}

Figure 11 shows the dependence of overall stress-strain relations on the maximum separation strength $T_{1} / \sigma_{\mathrm{Y}}$ with the grain size $d=0.1 l_{0}$. In this figure, we consider eight cases of $T_{1} / \sigma_{\mathrm{Y}}=0.5,1.0,2.0,3.0,4.0,6.0,8.0$ and 10.0. The results show that the overall strength and ductility of nc materials are very sensitive to the ratio $T_{1} / \sigma_{\mathrm{Y}}$. The tensile strength and ductility of nc materials increase with increasing of $T_{1} / \sigma_{\mathrm{Y}}$.

The relations of peak stress with $T_{1} / \sigma_{\mathrm{Y}}$ are shown in Fig. 12. The grain size effects in inelastic deformation are much bigger with increasing of $T_{1} / \sigma_{\mathrm{Y}}$. The dependence of intergranular fracture on the grain size is gradually diminished for grain diameter $d \geq l_{0}$, and the grain size effects in plastic flow are much bigger for $d=0.1 l_{0}$. The intragranular elastic-plastic deformation is dominant and the nc materials display a good ductility and high strength for the case of $T_{1} \geq \sigma_{\mathrm{Y}}$. On the 


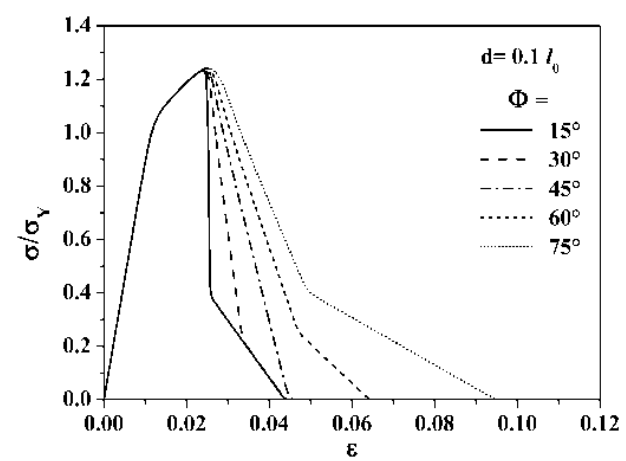

(a)

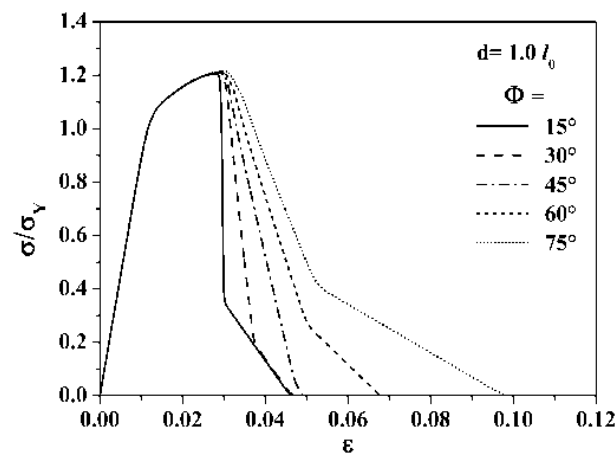

(b)

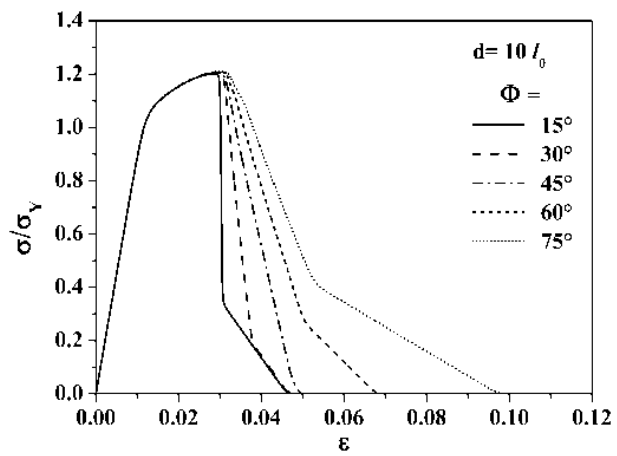

(c)

Fig. 8. Overall stress-strain curves for the several values of the ratio $G_{\mathrm{SC}} / G_{1 \mathrm{C}}$ (see Eq. (14)) (a)-(c) Correspond to three selected grain sizes.

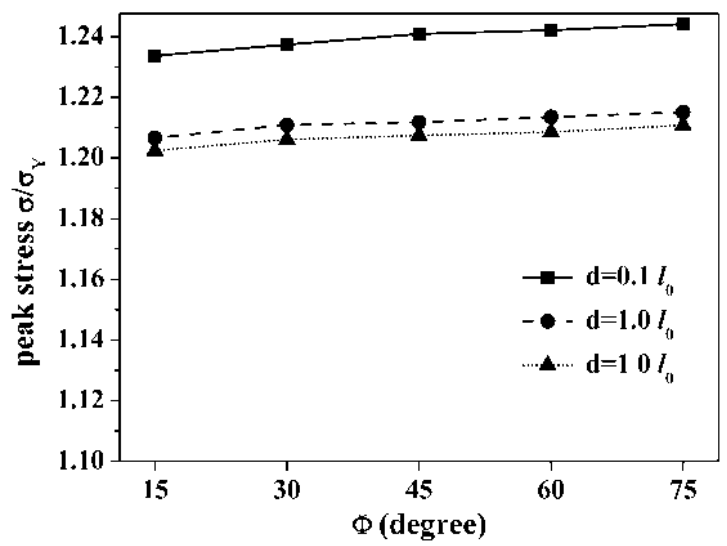

Fig. 9. The dependence of peak stress on the different ratio $G_{\mathrm{SC}} / G_{1 \mathrm{C}}$ for three selected grain sizes. 


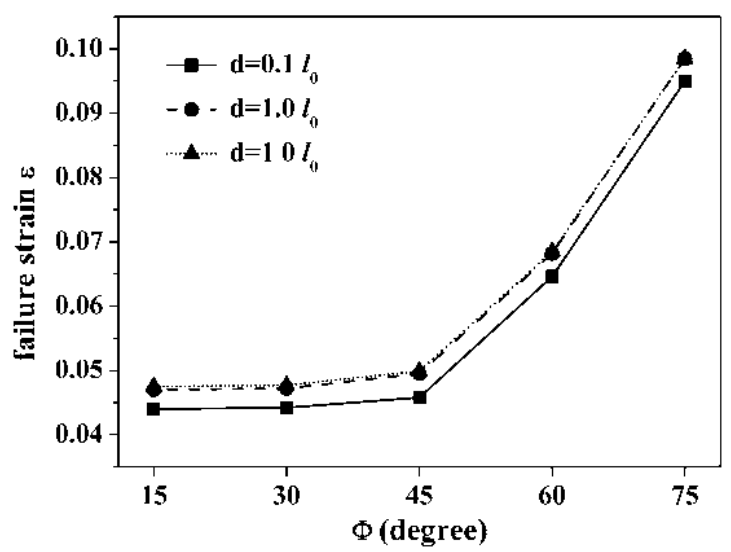

Fig. 10. The dependence of ultimate failure strain on the different ratio $G_{\mathrm{SC}} / G_{1 \mathrm{C}}$ for three selected grain sizes.

other hand, the grain boundaries may be weakened and cause the overall strength decreased for $T_{1}<\sigma_{\mathrm{Y}}$.

\subsection{Effect of fracture energy $G_{1 \mathrm{C}}$}

Figure 13 shows how the variation of fracture energy $G_{1 \mathrm{C}}$ influences the overall stress-strain relations for the grain size $d=0.1 l_{0}$. From Fig. 13, both the ductility and strength undergo an obvious increase. Figures 14 and 15 show the variations of peak stress and failure strain with normalized fracture energy $G_{1 \mathrm{C}}$ respectively. A comparison between Figs. 14 and 15 indicates that the ductility undergoes a more obvious increase with increasing the fracture energy $G_{1 \mathrm{C}}$.

\subsection{Comparison with experimental measurements}

In order to verify the validity of the present model, we use our model to simulate some cases where the experimental investigations were performed. The first experiment is on the nc $\mathrm{Cu}$ under uniaxial tension was reported in Sanders et al. [1997], and the second experiment on nc Ni under uniaxial tension was reported in Zhu et al. [2005].

In our simulation, the parameters of grain interior and the grain boundary for nc $\mathrm{Cu}$ and nc Ni are listed in Tables 1 and 2, respectively.

The grain boundary strength parameter $T_{1}$ is chosen to coincide with the yield strength $\sigma_{\mathrm{Y}}$, while grain boundary parameter $T_{\mathrm{S}}$ is generally selected in the range from yield strength $\sigma_{\mathrm{Y}}$ to shear strength for fcc metals, which is usually estimated as $\mu / 30$ ( $\mu$ is the shear modulus) [Wei and Anand, 2004]. For normal separation case such as in Wu and Wei [2010], one only needs to consider $T_{1}$, without $T_{\mathrm{S}}$, but for the present mixed mode we need to consider effects from both $T_{\mathrm{S}}$ (or $T_{1}$ ) and $T_{\mathrm{S}} / T_{1}$. 


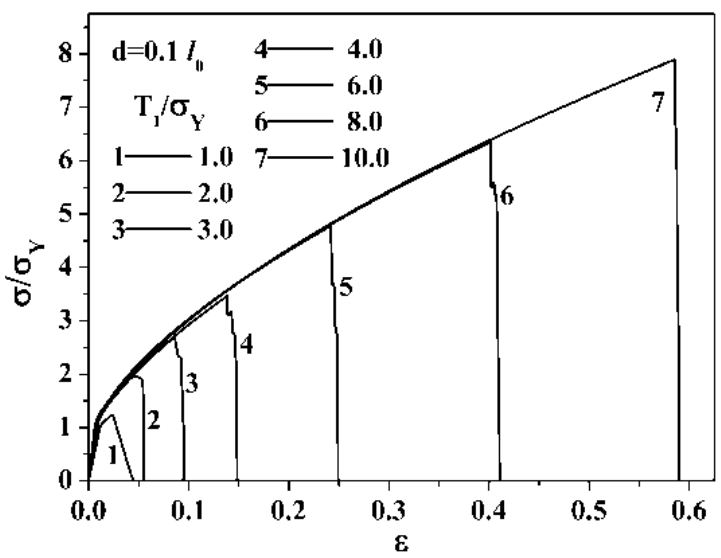

(a)

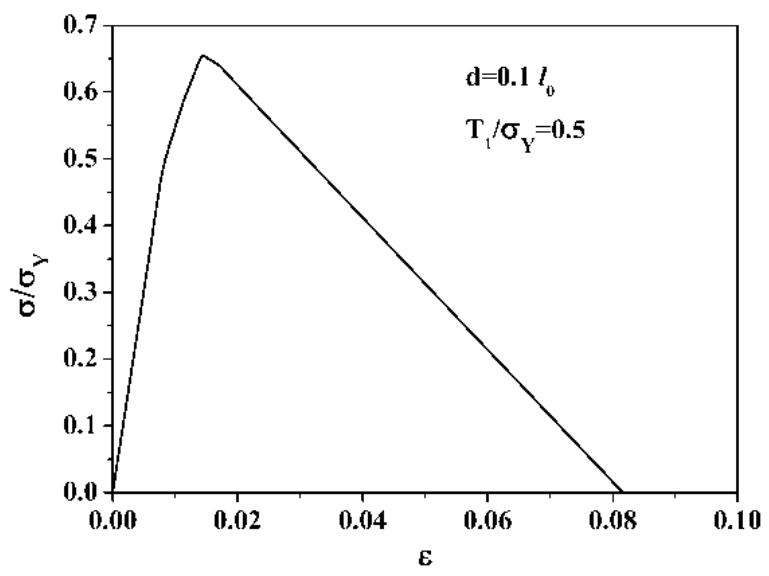

(b)

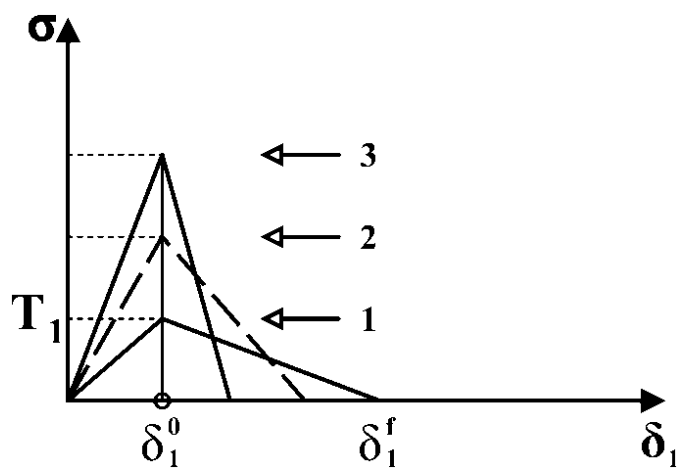

(c)

Fig. 11. Overall stress-strain curves for several values of the grain boundary separation strengths, $T_{1} / \sigma_{\mathrm{Y}}$. 


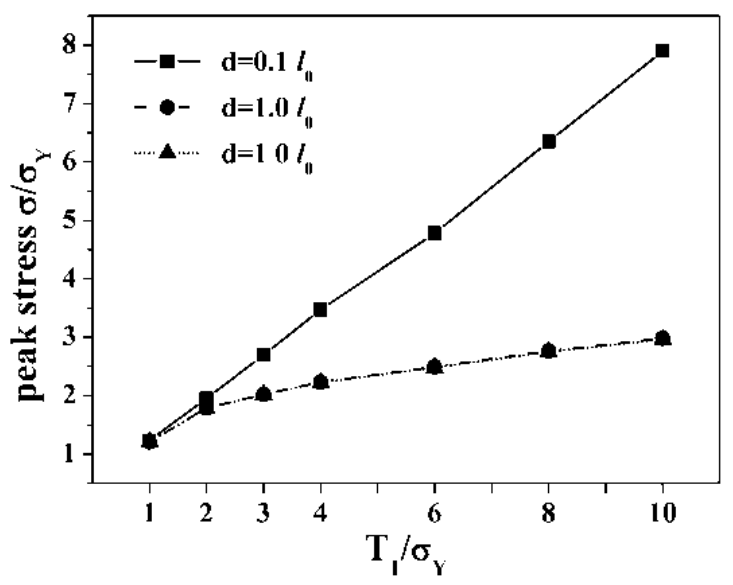

Fig. 12. The dependence of peak stress on different maximum separation strength of grain boundary $T_{1}$, for three selected grain sizes.

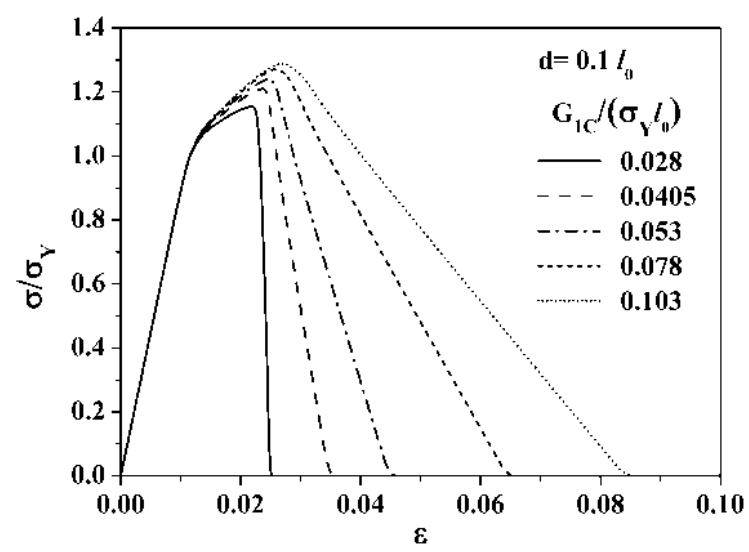

(a)

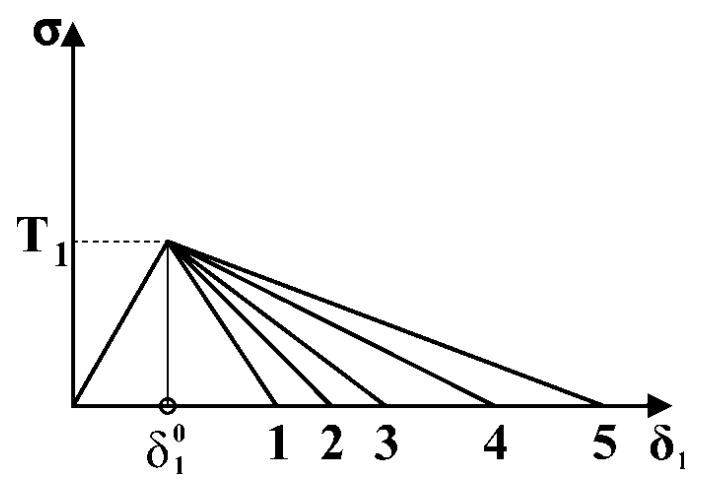

(b)

Fig. 13. Overall stress-strain curves for several values of the fracture energy $G_{1 \mathrm{C}}$. 


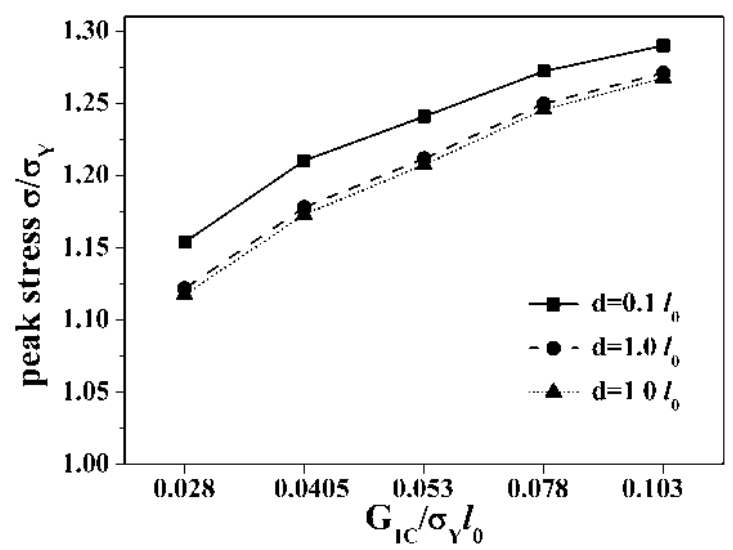

Fig. 14. The dependence of peak stress on different fracture energy $G_{1 \mathrm{C}}$ for three selected grain sizes.

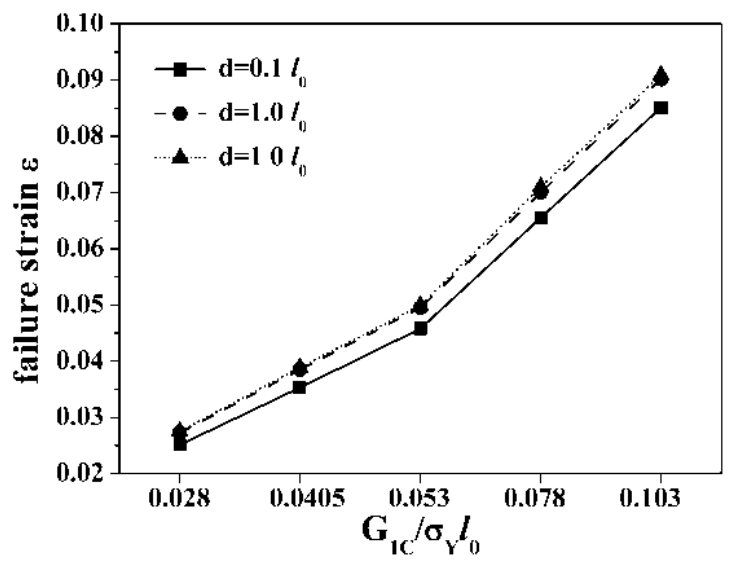

Fig. 15. The dependence of ultimate failure strain on different fracture energy $G_{1 \mathrm{C}}$ for three selected grain sizes.

Table 1. Simulation parameters for grain of nc metals, $\mathrm{Cu}$ and $\mathrm{Ni}$.

\begin{tabular}{cccccc}
\hline $\mathrm{Nc}$ metal & $E(\mathrm{GPa})$ & $v$ & $b(\mathrm{~nm})$ & $d(\mathrm{~nm})$ & $\sigma_{\mathrm{Y}}(\mathrm{GPa})$ \\
\hline $\mathrm{Cu}$ & 134 & 0.35 & 0.25 & 49 & 0.24 \\
$\mathrm{Ni}$ & 210 & 0.3 & 0.3 & 20 & 1.2 \\
\hline
\end{tabular}

Table 2. Simulation parameters for mixed-mode cohesive interface of nc metals, $\mathrm{Cu}$ and $\mathrm{Ni}$.

\begin{tabular}{ccccc}
\hline $\mathrm{Nc}$ metal & $T_{1}(\mathrm{GPa})$ & $T_{\mathrm{S}}(\mathrm{GPa})$ & $G_{1 \mathrm{C}}\left(\mathrm{J} / \mathrm{m}^{2}\right)$ & $G_{\mathrm{SC}}\left(\mathrm{J} / \mathrm{m}^{2}\right)$ \\
\hline $\mathrm{Cu}$ & 0.24 & 1.5 & 80 & 80 \\
$\mathrm{Ni}$ & 1.2 & 1.5 & 30 & 30 \\
\hline
\end{tabular}




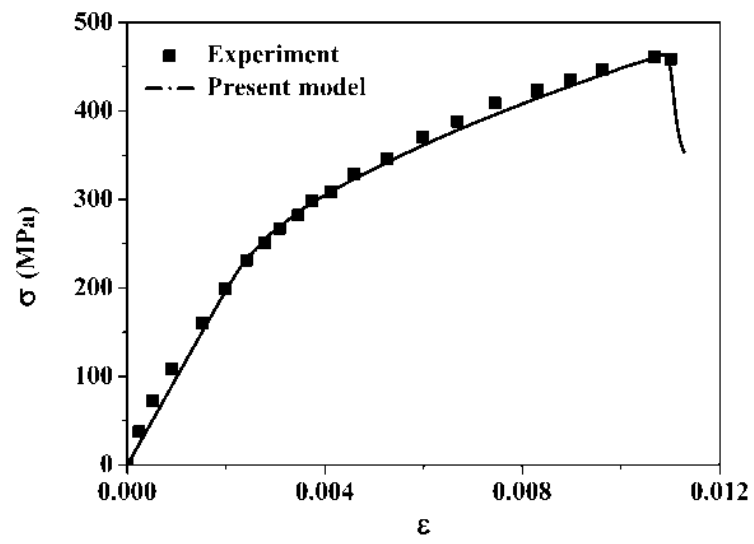

Fig. 16. Comparison of results (overall stress-strain relation) between present model and the experimental data for $\mathrm{nc} \mathrm{Cu}$.

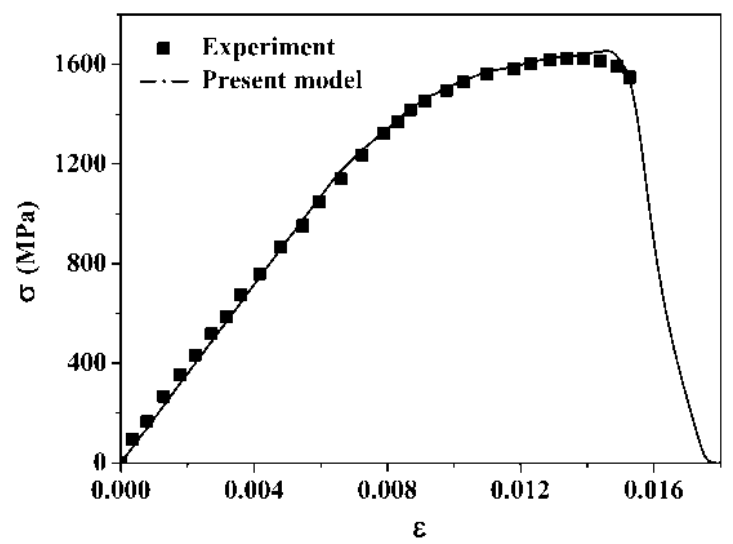

Fig. 17. Comparison of results (overall stress-strain relation) between our model and the experimental data for nc Ni.

Figures 16 and 17 show the comparisons between our model results and the corresponding experimental data for $\mathrm{nc} \mathrm{Cu}$ and $\mathrm{nc} \mathrm{Ni}$, respectively. From figures, the predicted stress-strain curves maintain a good agreement with the experimental data under tension.

The present simulations for the experiments confirm that the present model and FEM method developed for the nc materials are effective. The model and the numerical method can be used to describe the mechanical behavior of nc metals effectively.

\section{Concluding Remarks}

Through the systematical parametric studies on the size effect and overall mechanical behavior of nc materials, we conclude that five important parameters $\left(d, T_{1}\right.$, 
$G_{1 \mathrm{C}}, T_{\mathrm{S}} / T_{1}$ and $\left.G_{\mathrm{SC}} / G_{1 \mathrm{C}}\right)$, i.e., the grain size, critical separation strength, interface separation energy release rate, the mixity of separation strength, as well as the mixity of separation energy release rate, have the important influence on the size effect, strength and ductility for the nc materials. The effects of the five parameters on the size effect and overall mechanical behavior cause the transition of grain boundaries deformation with that of grain interiors at the different scale. Both $T_{1}$ and $T_{\mathrm{S}} / T_{1}$ jointly control the intragranular elastic-plastic deformation, while both $G_{1 \mathrm{C}}$ and $G_{\mathrm{SC}} / G_{1 \mathrm{C}}$ jointly control the grain boundary separation.

Through comparison of modeling and simulation with experiment, one can find that present model is convinced and can be used to characterize the overall mechanical behavior of nc metals effectively.

Usually, the grain boundary fracture processes were characterized by the cohesive zone model in the mechanical behavior simulations based on mechanics theory (in the present research corresponds to mixed-mode cohesive zone model), therefore the grain boundary properties of the nc materials are fully described by the parameters of the cohesive zone model. The higher-order effect of strain gradient within the grain boundary zone may be adjusted by selecting the cohesive interface parameters, and further work is needed to be done in the future. The strain gradient effects within interior grain are closely connected with grain size, and increase with decreasing the grain size, the simulation results of mechanical behavior depend on the normalized length scale (length scale of strain gradient theory over grain size).

Under the assumptions of the periodic boundary conditions for the nc materials, the simulation results of micro-structure evolutions are still of periodic characteristic, requiring the same micro-structure evolutions at neighboring cells, even though the grain boundary starts cracking and the cell failure occurs. This is a limitation of the periodic boundary condition assumptions that may be away from the true cases.

\section{Acknowledgment}

This work was supported by National Basic Research Program of China through 2012CB937500, and by the National Natural Science Foundation of China through Grants 11021262, 10932011 and 91116003. The first author (L.C.) would like to thank the helpful advice of Dr. Wei $\mathrm{Xu}$ from Institute of Mechanics, Chinese Academy of Sciences.

\section{References}

Barenblatt, G. I. [1959] "The formation of equilibrium cracks during brittle fracture. General ideas and hypothesis. Axially-symmetric cracks," Prikl. Matem. I Mekham 23, 434-444.

Barenblatt, G. I. [1962] "Mathematical theory of equilibrium cracks in brittle fracture," Advances in Applied Mechanics 7, 55-125.

Cao, A. and Wei, Y. [2007] "Atomistic simulations of crack nucleation and intergranular fracture in bulk nanocrystalline nickel," Physical Review B 76, 024113(5). 
Dugdale, D. S. [1960] "Yielding of steel sheets containing slits," Journal of the Mechanics and Physics of Solids 8, 100-104.

$\mathrm{Fu}$, H. H., Benson, D. J. and Meyers, M. A. [2004] "Computational description of nanocrystalline deformation based on crystal plasticity," Acta Materialia 52, 4413-4425.

Gao, H., Huang, Y., Nix, W. D. and Hutchinson, J. W. [1999] "Mechanism-based strain gradient plasticity - I. Theory," Journal of the Mechanics and Physics of Solids 47, $1239-1263$.

Gleiter, H. [2000] "Nanostructured materials: Basic concepts and microstructure," Acta Materialia 48, 1-29.

Hasnaoui, A., Van Swygenhoven, H. and Derlet, P. M. [2003] "Dimples on nanocrystalline fracture surfaces as evidence for shear plane formation," Science 300, 1550-1552.

Huang, Y., Qu, S., Hwang, K. C., Li, M. and Gao, H. [2004] "A conventional theory of mechanism-based strain gradient plasticity," International Journal of Plasticity 20, 753-782.

Hutchinson, J. W. [1976] "Bounds and self-consistent estimates for creep of polycrystalline materials," Proceedings of the Royal Society of London A 348, 101-127.

Kok, S., Beaudoin, A. J. and Tortorelli, D. A. [2002] "A polycrystal plasticity model based on the mechanical threshold," International Journal of Plasticity 18, 715-741.

Kumar, K. S., Van Swygenhoven, H. and Suresh, S. [2003] "Mechanical behavior of nanocrystalline metals and alloys," Acta Materialia 51, 5743-5774.

Mi, Y., Crisfield, M. A., Davies, G. A. O. and Hellweg, H.B. [1998] "Progressive delamination using interface elements," Journal of Composite Materials 32, 1246-1272.

Needleman, A. [1990] "An analysis of tensile decohesion along an interface," Journal of the Mechanics and Physics of Solids 38, 289-324.

Ovid'ko, I. A. [2007] "Review on the fracture processes in nanocrystalline materials," Journal of Materials Science 42, 1694-1708.

Sanders, P. G., Eastman, J. A. and Weertman, J. R. [1997] "Elastic and tensile behavior of nanocrystalline copper and palladium," Acta Materialia 45, 4019-4025.

Schiotz, J., Vegge, T., Di Tolla, F. D. and Jacobsen, K. W. [1999] "Atomic-scale simulations of the mechanical deformation of nanocrystalline metals," Physical Review B 60, $11,971-11,983$.

Schwaiger, R., Moser, B., Dao, M., Chollacoop, N. and Suresh, S. [2003] "Some critical experiments on the strain-rate sensitivity of nanocrystalline nickel," Acta Materialia $\mathbf{5 1 , 5 1 5 9 - 5 1 7 2 .}$

Shan, Z. W., Stach, E. A., Wiezorek, J. M. K., Knapp, J. A., Follstaedt, D. M. and Mao, S. X. [2004] "Grain boundary-mediated plasticity in nanocrystalline nickel," Science 305, 654-657.

Swaddiwudhipong, S., Tho, K. K., Hua, J. and Liu, Z. S. [2006] "Mechanism-based strain gradient plasticity in C-0 axisymmetric element," International Journal of Solids and Structures 43, 1117-1130.

Turon, A., Camanho, P., Costa, J. and Davila, C. [2004] "An interface damage model for the simulation of delamination under variable-mode ratio in composite materials," NASA/TM-2004-213277.

Tvergaard, V. and Hutchinson, J. W. [1992] "The relation between crack growth resistance and fracture process parameters in elastic-plastic solids," Journal of the Mechanics and Physics of Solids 40, 1377-1397.

Van der Sluis, O., Schreurs, P. J. G. and Meijer, H. E. H. [2001] "Homogenisation of structured elastoviscoplastic solids at finite strains," Mechanics of Materials 33, 499-522. 
Van Swygenhoven, H. and Derlet, P. A. [2001] "Grain-boundary sliding in nanocrystalline fcc metals," Physical Review B 64, 224105(9).

Van Vliet, K. J., Tsikata, S. and Suresh, S. [2003] "Model experiments for direct visualization of grain boundary deformation in nanocrystalline metals," Applied Physics Letters 83, 1441-1443.

Warner, D. H., Sansoz, F. and Molinari, J. F. [2006] "Atomistic-based continuum investigation of plastic deformation in nanocrystalline copper," International Journal of Plasticity 22, 754-774.

Wei, Y. G. and Hutchinson, J. W. [1997a] "Steady-state crack growth and work of fracture for solids characterized by strain gradient plasticity," Journal of the Mechanics and Physics of Solids 45, 1253-1273.

Wei, Y. G. and Hutchinson, J. W. [1997b] "Nonlinear delamination mechanics for thin films," Journal of the Mechanics and Physics of Solids 45, 1137-1159.

Wei, Y. J. and Anand, L. [2004] "Grain-boundary sliding and separation in polycrystalline metals: Application to nanocrystalline fcc metals," Journal of the Mechanics and Physics of Solids 52, 2587-2616.

Wei, Y. G., Shu, S. Q., Du, Y. and Zhu, C. [2005] "Size, geometry and nonuniformity effects of surface-nanocrystalline aluminum in nanoindentation test," International Journal of Plasticity 21, 2089-2106.

Wei, Y. [2006] "A new finite element method for strain gradient theories and applications to fracture analyses," European Journal of Mechanics A - Solids 25, 897-913.

Wei, Y., Chen, X., Shu, S. and Zhu, C. [2006] "Nonuniformity effect of surfacenanocrystalline materials in nanoindentation test," International Journal for Multiscale Computational Engineering 4, 183-195.

$\mathrm{Wu}$, B. and Wei, Y. G. [2010] "A computational model for intergranular fracture in nanocrystalline and ultra-fine polycrystalline metals," Materials Science Forum 633634, 39-53.

Wu, B., Liang, L. H., Ma, H. S. and Wei, Y. G. [2012] "A trans-scale model for size effects and intergranular fracture in nanocrystalline and ultra-fine polycrystalline metals," Computational Materials Science 57, 2-7.

$\mathrm{Xu}, \mathrm{W} ., \mathrm{Lu}, \mathrm{T}$. J. and Wang, F. [2010] "Effects of interfacial properties on the ductility of polymer-supported metal films for flexible electronics," International Journal of Solids and Structures 47, 1830-1837.

Zhu, B., Asaro, R. J., Krysl, P. and Bailey, R. [2005] "Transition of deformation mechanisms and its connection to grain size distribution in nanocrystalline metals," Acta Materialia 53, 4825-4838. 